\title{
Hybrid Molecular Mechanics: For Effective Crystal Field Method for Modeling Potential Energy Surfaces of Transition Metal Complexes
}

\author{
M. B. DARHOVSKII, ${ }^{1,2}$ M. G. RAZUMOV, ${ }^{3}$ I. V. PLETNEV, ${ }^{2,4}$ \\ A. L. TCHOUGRÉEFF ${ }^{\mathbf{1}, 2}$ \\ ${ }^{1}$ L. Y. Karpov Institute of Physical Chemistry, Moscow, Russia \\ ${ }^{2}$ Center for Computational Chemistry at the M. V. Keldysh Institute for Applied Mathematics of \\ RAS, Moscow, Russia \\ ${ }^{3}$ N. S. Kurnakov Institute of General and Inorganic Chemistry of RAS, Moscow, Russia \\ ${ }^{4}$ Chemistry Department, M. V. Lomonosov Moscow State University, Moscow, Russia
}

Received 20 July 2001; accepted 22 December 2001

DOI 10.1002/qua.10211

\begin{abstract}
The effective crystal field (ECF) methodology previously developed for description of the electronic structure of transition metal complexes (TMCs) is combined with molecular mechanics (MM) formalism. In this way, a new method for calculations of potential energy surfaces of the Werner-type TMC is developed. It is based on a combined quantum mechanics (QM)-MM approach with the ECF method taking part of the QM and advanced MM package MMPC developed for the metal ion complex computations and based on the CHARMM organic force field. The MM region consists of ligand atoms and metal ion coordination sphere, leaving out effects of $d$-shell, while the QM region is limited to metal ion $d$-shell. Crystal field matrix for the $d$-shell is calculated with use of the local ECF method. It is shown that the procedure proposed reproduces with considerable accuracy geometry characteristics of values of the Fe(II) complexes with both mono- and polydentate ligands. (c) 2002 Wiley Periodicals, Inc. Int J Quantum Chem 88: 588-605, 2002
\end{abstract}

Key words: effective crystal field; transition metal complexes; potential energy surfaces; quantum mechanics/molecular mechanics

\section{Introduction}

M olecular mechanics (MM) [1] is widely used in calculations of potential energy surfaces (PESs) of organic molecules, both per se and in

\footnotetext{
Correspondence to: A. L. Tchougréeff; e-mail: andrei@cc.nifhi. ac.ru.
}

molecular dynamics simulations. The conventional MM scheme faces, however, significant problems when applied to the metal ion complexes. The main problem is an adequate modeling of coordination sphere, which, in fact, presents two closely related subproblems: accounting for coordination polyhedron flexibility and for electronic structure details.

Conventional MM considers metal center (as well as all the other atoms) as having a definite 
ideal (strain-free) stereochemistry, which is allowed to be distorted only slightly. Electronic structure is accounted for only implicitly, through the choice of ideal polyhedron and associated with it deformation potentials/constants. The typical paradigm, valence force field with harmonic potentials (VFF$\mathrm{H})$, requires definition of a unique set of ideal bond lengths and valence angles around the metal ion and associated stretching/bending constants. For most metal ions, the VFF-H model prevents analysis of real-world, significantly distorted structures, as well as structural rearrangements (which are numerous in coordination chemistry).

The obvious solution of the problem is to reject the VFF-H formalism for metal ions, in parts or at all. The most straightforward way is to describe (angular) deformations in coordination sphere with specially dedicated potential functions rather than with harmonic potentials. In this respect, a moderately successful use of trigonometric or double-well potentials has been reported [2-5]. A more radical and popular approach is the so-called "points-ona-sphere" (POS) scheme [6-9]. It suggests that the shape of coordination polyhedron is ultimately dictated by the ligand-ligand van der Waals interactions, thus eliminating the need in definition of idealized polyhedron and associated angular parameters. Recently, it has been shown $[10,11]$ that this approach may be further improved by considering not van der Waals interligand interactions but "coordination bonds repulsion," as suggested by well-known and extremely successful qualitative theories by Gillespie and Hargittai [12] and Kepert [13]. To our mind, this last version of MM, so-called Gillespie-Kepert MM (MMGK) [10, 11] is a maximum of what may be reached within the pure MM framework. It allows a proper description of many cases of significant distortion in coordination geometry (for a discussion and examples, see Refs. [10, 11]).

However, even this last MMGK version of MM suffers from a general problem of being not capable to account for important consequences of details of electronic structure of transition metal ions (TMIs). This is not a technical issue that may be solved by use of more intricate versions of potential functions. As mentioned in Ref. [14], the physical precondition of successful use of MM for common organic molecules is that their electronic excited states are well separated from the respective ground states on the energy scale. Only one quantum state of electronic system is experimentally observed and the MM (a sort of classic) description becomes valid. By contrast, the behavior of the metal valence $d$-shell is sufficiently quantum: Several electronic states may appear in a narrow energy range close to its ground state so that sometimes the PESs corresponding to different electronic terms of the $d$-Shell intersect, resulting in spin transitions $[15,16]$.

Consequently, it seems necessary to directly incorporate a quantum mechanical (QM) description of the central atom and its closest surrounding into computational scheme while the rest of molecule may be still described within MM formalism. In other words, we need hybrid quantum mechanics/ molecular mechanics (QM/MM) methods.

Recently, a general approach to description of the molecular electronic structure and potential energy has been suggested [17] that makes it possible to apply quantum chemical description to that part of molecule in which electronic terms are close on the energy scale and use the MM description for the part where the closest electronic states remain distant in energy. An analogous approach has been also applied in articles [14, 18] for the PES evaluation of the spin-crossover in Fe(II) complexes. It was suggested in Ref. [14] to calculate the energy of the $d$-shell as that of the ground state of the $d^{n}$-configuration in the effective crystal field (ECF; see below) induced by the ligands. The energy of the $d$-shell in the ECF approximation depends both on the molecular geometry (position and orientation of the ligands around the central ion) and electronic structure of the ligands. However, the approach [14] is computationally intensive as it requires calculation of electronic structure of the whole ligand sphere at each step of geometry optimization, which reduces its practical significance [14, 18].

Another approach to incorporating TMI in the general MM scheme was proposed in Refs. [19, 20]. It uses the angular overlap model (AOM) in the cellular ligand field (CLF) formulation [21] and represents the energy of the $d$-shell in the crystal field as a sum of one-electron energies of the occupied $d$-levels in a one-electron approximation. The crystal field acting upon the $d$-states, in turn, is represented as a sum of contributions from each ligand, described by the AOM parameters $e_{\bar{\sigma}}$ and $e_{\pi}$. Dependence of the AOM parameters on metal-ligand distance in Refs. $[19,20]$ is interpolated by fitting empirical values of the former for different ligands. This approach (CLFSE/MM, [19]) has been used for calculation of the structures of nickel (II) complexes with amine and $\pi$-type ligands. However, CLFSE neglects the $d$-electron correlations which, in fact, are responsible for the form of the energy low- 
excitation spectrum of the $d$-shell. Consequently, the ECF scheme seems to be of more general applicability (see Discussion).

In this article, we report a computationally efficient combination of the local [22] version of the ECF method-LECF with the MMGK procedure and the application of the hybrid method to the calculations of molecular geometry for a series of $\mathrm{Fe}$ (II) complexes (having different ground-state total spin) with nitrogen-containing ligands.

The article is organized as follows: In the next section, we briefly review the basic features of the ECF method [23] and of its local incarnation [22]. Next, we describe a new approach allowing us to determine the ECF as a sum of contributions from the separate ligands taking into account the chemical nature of the ligands and (with use of perturbation technique) the Coulomb interaction of the ligand with the rest of the complex. The last section provides the examples of application of ECF/ MMGK to some real-world problems.

\section{Theory: Estimation of Effective Crystal Field}

As mentioned in the Introduction, the key point for incorporation of TMI into MM is to estimate the energy of the $d$-shell as a function of the ligand sphere geometry and composition. In this section, we develop the working formulae based on the ECF theory performing this task.

\section{BASICS OF THE ECF}

The ECF method is based on a general concept of separating electron variables. In the context of the original ECF method [23], the motivation of such a separation is that the electron correlations are much more significant in the $d$-shell rather than in the ligands and for that reason different levels of approximation must be used for different parts of the TMC. The same concept applies when a hybrid $\mathrm{QM} / \mathrm{MM}$ method is to be developed [24]: Electrons have to be divided into groups; some of the groups whose excited electronic states are available in the experiment are treated quantum mechanically whereas the behavior of other groups whose excited electronic states lay high in energy (and are not available in experiment) are modeled with use of MM. In a TMC containing one transition metal ion and ligands around it, the separation of elec- trons into groups is performed as follows: The basis of valence atomic orbitals (AOs) containing the 4s-, $4 p-$, and $3 d-\mathrm{AOs}$ of the metal atom (for a firsttransition-row element) and those of the ligand atoms is, according to Ref. [23], divided into two parts. The first contains only $3 d$-orbitals of the transition metal atom ( $d$-system). The rest contains $4 s^{-}$, $4 p$-AOs of the metal atom and the valence AOs of the ligand atoms. All these orbitals together are termed as ligand (l-)system. In the ECF method [23], it is shown that the $d$-shell can be described by the effective QM Hamiltonian $H_{d}^{\text {eff. }}$

$$
H_{d}^{\mathrm{eff}}=\sum_{\mu \nu \sigma} U_{\mu \nu}^{\mathrm{eff}} d_{\mu \sigma}^{+} d_{\nu \sigma}+\frac{1}{2} \sum_{\mu \nu \rho \eta} \sum_{\sigma \tau}(\mu \nu \mid \rho \eta) d_{\mu \sigma}^{+} d_{\rho \tau}^{+} d_{\eta \tau} d_{\nu \sigma}
$$

where the $d$-electron Coulomb interaction term is inherited from the free ion and the effective core parameters $U_{\mu \nu}^{\text {eff }}$ contain contributions from the Coulomb and the resonance interaction between the $d$ - and $l$-systems:

$$
U_{\mu \nu}^{\mathrm{eff}}=\delta_{\mu \nu} U_{d d}+W_{\mu \nu}^{\mathrm{atom}}+W_{\mu \nu}^{\mathrm{field}}+W_{\mu \nu}^{\mathrm{cov}},
$$

where

$$
W_{\mu \nu}^{\text {atom }}=\delta_{\mu \nu}\left(\sum_{\alpha \in s, p} g_{\mu \alpha} P_{\alpha \alpha}\right)
$$

is the repulsion of electrons in the $d$-shell from those in the $4 s$ - and $4 p$-AOs of the metal;

$$
W_{\mu \nu}^{\text {field }}=\sum_{L} Q_{L} V_{\mu \nu}^{L}
$$

is the Coulomb interaction of $d$-electrons with the net charges on the ligand atoms, having the standard crystal field theory form [25]; and the covalence part:

$$
W_{\mu \nu}^{\mathrm{cov}}=-\sum_{i} \beta_{\mu i} \beta_{v i}\left(\frac{1-n_{i}}{\Delta E_{d i}}-\frac{n_{i}}{\Delta E_{i d}}\right)
$$

ultimately comes from the resonance interaction between the $d$ - and $l$-systems. According to the ECF method [23], the l-system is described by a single Slater determinant $\Phi_{l}$ that has to be obtained from a self-consistent field (SCF) procedure. Solving the SCF problem for the $l$-system allows us to deter- 
mine one-electron density matrix $P_{\alpha \beta}$, orbital energies of molecular orbitals (MOs) $\epsilon_{i}$, and the MOLCAO coefficients $c_{i \alpha}$. These quantities completely define the electronic structure of the $l$-system and are used to calculate the effective Hamiltonian (1) because in Eqs. (3)-(5) $Q_{L}=\sum_{\alpha \in L} P_{\alpha \alpha}-Z_{L}$ is the effective charge of the ligand atom $L ; Z_{L}$ is the core charge of the ligand atom $L ; V_{\mu \nu}^{L}$ is the matrix element of the potential energy operator describing the interaction between a $d$-electron and a unit charge placed on the ligand atom $L ; n_{i}$ is the occupation number of the $i$ th $l-\mathrm{MO}\left(n_{i}=0\right.$ or 1$) ; \Delta E_{d i}$ $\left(\Delta E_{i d}\right)$ is the energy necessary to transfer an electron from the $d$-shell (from the $i$ th $l-\mathrm{MO}$ ) to the $i$ th $l-\mathrm{MO}$ (to the $d$-shell):

$$
\begin{aligned}
& \Delta E_{d i}=-A_{i}+I_{d} \\
& \Delta E_{i d}=I_{i}-A_{d},
\end{aligned}
$$

where $I_{i}$ and $A_{i}$ are the ionization potential and the electron affinity of the $i$ th $l-\mathrm{MO}$ and $I_{d}$ and $A_{d}$ are, respectively, the effective ionization potential and the electron affinity of the $d$-shell. The resonance integrals $\beta_{\mu i}$ in Eq. (5) are given by

$$
\beta_{\mu i}=\sum_{\alpha} \beta_{\mu \alpha} c_{i \alpha}
$$

where $c_{i \alpha}$ is the MO-LCAO coefficient and $\beta_{\mu \alpha}$ is the resonance integral between the $\alpha$ th $l-\mathrm{AO}$ and the $\mu$ th $d$-AO.

\section{LOCAL VERSION OF ECF}

As one can see the ECF method [23] involves the covalent term Eq. (5) to the crystal field, which is strongly related to the canonical MOs (CMOs) of the l-system. According to calculations [23, 26-29], namely, the covalent term dominates (up to 80$90 \%$ ) the $d$-level splitting. The Coulomb interaction with the effective charges on the ligand atoms gives the remaining $10-20 \%$ of the splitting and incidentally has local character. The AOM method employed in Refs. [19, 20], by contrast, operates with some local quantities, which are, however, not clearly defined and cannot be obtained in another way rather than fitting experimental data [21, 30]. The interpolation for the AOM parameters proposed and used in Refs. $[19,20]$ in the framework of the CLFSE approach is too much simplified in two respects: (1) It does not reflect the chemical identity of the ligand because the single formula (see below) is used for all ligands of similar structure and composition, although the AOM theory itself [21, 30] insists on the chemical specificity of its parameters; (2) the dependence of the AOM parameters on molecular geometry adopted in Refs. $[19,20]$ does not cover that on orientation of the ligands with respect to the metal atom.

In our recent article [22], we derived and tested a local version of the ECF method and calculated the $\mathrm{AOM}$ parameters on its basis. Here we review briefly the derivarion of the local form for the covalent part of the ECF $W_{\mu \nu}^{\mathrm{cov}}$, which allows us to recast it into the form compatible with the MM approach and reformulate the calculation of the $d$-shell energy in terms of the AOM parameters, which now become calculable for arbitrary geometry of the ligand sphere and absorb the information on the electronic structure of the ligands (see below). To this end, we notice that Eq. (5) can be rewritten in terms of the retarded (ret) and advanced (adv) Green's functions for the l-system [31, 32]:

$$
\begin{aligned}
G_{i i}^{\mathrm{ret}}(z) & =\left\langle\Phi_{l}\left|a_{i}\left(F_{l}^{\mathrm{eff}}-z\right)^{-1} a_{i}^{+}\right| \Phi_{l}\right\rangle \\
G_{i i}^{\mathrm{adv}}(z) & =-\left\langle\Phi_{l}\left|a_{i}^{+}\left(F_{l}^{\mathrm{eff}}-z\right)^{-1} a_{i}\right| \Phi_{l}\right\rangle
\end{aligned}
$$

taken at $z=I_{d}, A_{d}$, respectively. (Here, $F_{l}^{\text {eff }}$ is the effective one-electron Fock operator for the $l$-system.) Poles of the $l$-system Green's function are the ionization potentials $I_{i}$ and electron affinities $A_{i}$ of the latter related to the $i$ th $l-\mathrm{MO}$, whether occupied or vacant, respectively. Taking into account that the removed (or added) electron is actually transferred to (from) the transition metal atom, rather than to (from) infinity, the ionization potential and electron affinity of the $l$-system acquire the form:

$$
\begin{gathered}
I_{i}=-\boldsymbol{\epsilon}_{i}-g_{d i} \\
A_{i}=-\boldsymbol{\epsilon}_{i}+g_{d i r}
\end{gathered}
$$

where $g_{d i}$ is the energy of the Coulomb interaction between an electron and a hole that are located in the metal $d$-shell and on the $i$ th $l$-MO, respectively.

Then, for Eq. (5) we get

$$
W_{\mu \nu}^{\mathrm{cov}}=-\sum_{i} \beta_{\mu i} \beta_{v i}\left[G_{i i}^{\mathrm{ret}}\left(I_{d}\right)+G_{i i}^{\mathrm{adv}}\left(A_{d}\right)\right] .
$$


The Green's functions allow us to express the result of Eq. (5) in the arbitrary basis of the oneelectron states of the $l$-system. We perform a unitary transformation of the $l$-system CMOs $|i\rangle$, which are the eigenstates of the respective Fock operator to the localized MOs (LMOs) $|L\rangle$ for the occupied and empty MOs separately:

$$
|L\rangle=\sum_{i} c_{i L}|i\rangle .
$$

Summation over $i$ in Eq. (9) runs either over occupied or vacant MO. Here, $c_{i L}$ are the coefficients of the $i$ th CMO in the expansion of the Lth LMO (the coefficients $c_{i L}$ are invariants of the molecular electronic structure and do not change under the coordinate frame rotation). The expansion of LMOs over the AOs has the form

$$
c_{L \alpha}=\sum_{i} c_{i L} c_{i \alpha}
$$

where $c_{L \alpha}$ is the coefficient of the $\alpha$ th $\mathrm{AO}$ in the expansion of the Lth LMO. There are many localization techniques known in the literature that are used to find the transformation coefficients $c_{i L}$. We used that based on the max $\Psi^{4}$ localization procedure [33], which is the most feasible technically. We modified the procedure built into the MOPAC6 package [34] to make it capable of performing localization of either occupied or vacant orbitals. The max $\Psi^{4}$ procedure results in strongly localized states. In the framework of the accepted localization method, the donor atom AOs give the main contribution to the LPs ( $\sim 93 \%$ from $s$ - and $p$-AO of the donor atom) whereas the metal $4 s-$ and $4 p$-AOs give $\sim 6 \%$. Contribution from other AOs does not exceed $1 \%$. Also, the LPs are well localized on the energy scale. In the case of the $\left[\mathrm{Fe}(\mathrm{py})_{6}\right]^{2+}$ molecule, all contributions to the LP of the pyridine ligand are located in four energy intervals having widths not exceeding 0.1 a.u., which give respectively in order of increasing energy 17, 17, 32, and 28\% (totally $94 \%)$ of the LP weight. For the $\left[\mathrm{Co}\left(\mathrm{NH}_{3}\right)_{6}\right]^{2+}$ and $\left[\mathrm{Fe}\left(\mathrm{H}_{2} \mathrm{O}\right)_{6}\right]^{2+}$ complexes, a similar picture is observed [22].

In the basis of the LMOs, the resonance integrals entering Eq. (5) acquire the form

$$
\beta_{\mu i}=\sum_{L} \beta_{\mu L} c_{i L}
$$

$$
\beta_{\mu L}=\sum_{\alpha} c_{L \alpha} \beta_{\mu \alpha} .
$$

Inserting the above $\beta_{\mu i} \mathrm{~s}$ in the expression for $W_{\mu \nu}^{\text {cov }}$ in Eq. (8) (further on the resonance integrals $\beta_{\mu i}$ and $\beta_{\mu L}$ refer, respectively, to the CMOs and LMOs basic sets) one gets

$$
\begin{aligned}
& W_{\mu \nu}^{\mathrm{cov}}=-\sum_{i} \beta_{\mu i} \beta_{\nu i}\left(\frac{1-n_{i}}{\Delta E_{d i}}-\frac{n_{i}}{\Delta E_{i d}}\right) \\
&=-\sum_{L L^{\prime}} \beta_{\mu L} \beta_{v L^{\prime}} \sum_{i} c_{i L^{\prime}} c_{i L^{\prime}}\left(\frac{1-n_{i}}{I_{d}-g_{d i}+\epsilon_{i}}\right. \\
&\left.-\frac{n_{i}}{-A_{d}-g_{d i}-\epsilon_{i}}\right) \\
&=\sum_{L L^{\prime}} \beta_{\mu L} \beta_{\nu L^{\prime}}\left\{G_{L L^{\prime}}^{\mathrm{ret}}\left(I_{d}\right)+G_{L L^{\prime}}^{\mathrm{adv}}\left(A_{d}\right)\right\} .
\end{aligned}
$$

Clearly, the unitary transformation from the CMOs basis to the LMOs basis of the $l$-system does not affect the covalent part of the ECF. It turned out, however (see Ref. [22]), that the sum over diagonal pairs with $L^{\prime}=L$ in (12) is the most efficient and convenient approximation for $10 \mathrm{Dq}$ with the error not exceeding $0.1 \mathrm{eV}$. This accuracy is also comparable to that of the ECF method itself. The formal expression for this approximate summation is

$$
W_{\mu \nu}^{\mathrm{cov}}=\sum_{\Lambda} \sum_{L \in \Lambda} \beta_{\mu L} \beta_{\nu L} G_{L L}^{\mathrm{adv}}\left(A_{d}\right),
$$

where the index $\Lambda$ enumerates the ligands where the LPs are located; the subscript $L$ is that of the LP itself. Because in the above formula only the LPs are employed that have contributions only from the occupied CMOs, Eq. (13) contains only the advanced Green's function. By this, we arrive to the local form of the ECF theory.

\section{PERTURBATIVE TREATMENT OF $L$-SYSTEM}

In the previous sections, we reviewed the ECF theory and its local reformulation (LECF), which allowed us to derive and calculate the crystal field. It is in particular important that by this method this can be done for arbitrary geometry of the complex, a prerequisite for developing a hybrid QM/MM method. It is, however, one-half way to the goal. Although the summation scheme over the local 
states in Eq. (13) is approximate, the Green's function of the $l$-system employed in it is still an exact one. It represents formally in the local basis the result of the complete SCF procedure applied to the whole $l$-system. It implicitly involves all the orbitals of the l-system, namely, the metal $4 s, 4 p$-AOs, and the valence orbitals of the ligands. It would be, however, more computationaly feasible to express the ECF matrix (and thus the energy of the $d$-shell) in terms of the electronic structure parameters of the free ligands. Toward this end, the Green's function of the $l$-system $G^{l}$ has to be expressed in terms of free ligands (or even of their fragments in the case of polydentate ligands with several donor atoms).

The natural way to do so is to apply the perturbation technique taking the Green's function of a system consisting of noninteracting metal ion and ligands as an unperturbed one. In general, two kinds of interactions between the central metal ion and the ligands must be turned on along a way from the state of isolated ligands and TMI to this bound state in the complex. The first is the Coulomb interaction of the ligands with the field of the central ion having the charge $\left(Z_{M}-n_{d}\right)$ e. The second is the resonance responsible for one-electron transfers between the ligands and the $4 s-, 4 p$ - AOs of the metal atom. The bare Green's function for the unperturbed $l$-system $\mathbf{G}_{00}^{l}$ has the following simple block diagonal form:

$$
\mathbf{G}_{00}^{l}=\left(\begin{array}{cccc}
\mathbf{G}_{0}^{M} & 0 & 0 & 0 \\
0 & \mathbf{G}_{0}^{\Lambda_{1}} & 0 & 0 \\
0 & 0 & \ddots & 0 \\
0 & 0 & 0 & \mathbf{G}_{0}^{\Lambda_{n}}
\end{array}\right)
$$

Nonvanishing blocks $\mathrm{G}_{0}^{\Lambda_{i}}$ correspond to separate ligands (fragments) $\Lambda_{i}$ containing the diagonal $G_{L L}^{\Lambda}(\epsilon)$ Green's function matrix elements of the lone pair $L$ at $\Lambda_{i}$ :

$$
\left(G_{0}^{\Lambda}(\epsilon)\right)_{L L}^{\mathrm{adv}}=\sum_{i \in \Lambda} \lim _{\delta \rightarrow 0^{+}} \frac{\left(c_{i L}^{\Lambda}\right)^{2}}{\epsilon-\epsilon_{i \Lambda}^{(0)}+i \delta^{\prime}}
$$

where summation involves all the MOs that have nonvanishing $c_{i L}^{\Lambda}$ contribution to the lone pair $L$; the off-diagonal matrix elements $G_{L L^{\prime}}^{\Lambda}$ are nonvanishing only if the LMOs $L$ and $L^{\prime}$ belong to the same ligand $\Lambda$ (fragment).

The Green's function matrix block $\mathrm{G}_{0}^{M}$ has the form

$$
G_{0}^{M}(\epsilon)_{m m^{\prime}}^{\mathrm{ret}}=\lim _{\delta \rightarrow 0^{+}} \frac{\delta_{m m^{\prime}}}{\epsilon-\epsilon_{m}^{(0)}-i \delta^{\prime}}
$$

where the subscript $m$ runs over $4 s$ and $4 p(x, y, z)$ AOs of the TMI; the energies $\epsilon_{m}^{(0)}$ correspond to those of the $m$ th level of the free metal ion, that is,

$$
\begin{aligned}
& \boldsymbol{\epsilon}_{4 s}^{(0)}=U_{s s}+n_{d} g_{s d} \\
& \boldsymbol{\epsilon}_{4 p}^{(0)}=U_{p p}+n_{d} \bar{g}_{p d},
\end{aligned}
$$

where $U_{p p}, U_{s s}$ and $g_{s d}, \bar{g}_{p d}$ are, respectively, parameters of the core attraction and repulsion of electrons placed in the $s$ - and $p$-orbitals of the metal atom from its $d$-electrons.

The two sorts of interactions mentioned above play, however, different roles. The Coulomb interaction does not break the block-diagonal structure of the bare Green's function $\mathbf{G}_{00}^{l}$. By contrast, the resonance interaction smashes the block structure. As shown in Refs. [22, 23, 26-29, 35], the total electron transfer from each ligand to the metal ion $4 s-, 4 p$-AOs amounts to $0.1-0.15$ of unit charge per ligand at the physically reasonable metal-ligand distances (at longer separations it is even smaller). This makes it possible to take the resonance into account with use of perturbation theory in the metalligand one-electron hopping. In this article, we restrict ourselves to the effect of the Coulomb field only, that is, to the zero order with respect to the resonance. In this setting, the LMOs of the $l$-system can be expanded in terms of the CMOs not of the whole $l$-system, but in terms of the MOs of the respective ligand (fragment) only. Thus, the summation index $i$ in the second row of Eq. (12) enumerates the MOs for each ligand (fragment) separately. The CMO orbital energies (forming narrow quasibands) that appear as the Green's function poles can be replaced by the close values of the orbital energies of the separate ligands (fragments) within the complex. That allows us to conserve the block-diagonal form also for the approximate Green's function for the $l$-system:

$$
\begin{aligned}
& \mathbf{G}_{0}^{l}=\mathbf{G}^{L} \oplus \mathbf{G}^{M} \\
& \mathbf{G}^{L}={ }_{\Lambda}^{\oplus} \mathbf{G}^{\Lambda},
\end{aligned}
$$

where $\mathbf{G}^{M}$ and $\mathbf{G}^{\Lambda}$ are the Green's function matrices $\mathrm{G}_{0}^{M}$ and $\mathrm{G}_{0}^{\Lambda}$ but with the poles (orbital energies) of the metal ion in the Coulomb field induced by the 
charges localized on the ligand atoms whereas the poles of Green's function matrices $\mathrm{G}^{\Lambda}$ correspond to the orbital energies of the ligand molecules in the Coulomb field induced by the central ion and by other ligands $\left(\Lambda^{\prime} \neq \Lambda\right)$ rather than to those of the free ligands.

In the following subsection, we consider an implementation of this approach allowing us to express the Green's function of the $l$-system in terms of the Green's functions of separate ligands taking into account the Coulomb field effects.

\section{EFFECTS OF COULOMB FIELD WITHIN THE RIGID LIGANDS' MOs MODEL}

The accepted model of electronic structure of TMC considers the metal ion as a point charge (equal to its oxidation degree or formal charge). The electron transfers from the ligand orbitals to the metal ion valence AOs are neglected. Within such an approach, the influence of the central ion on the surrounding ligands reduces to that of the Coulomb field. The Coulomb field affects the positions of the poles of the Green's function (orbital energies) of the free ligands. The form of the CMOs of each ligand remain unchanges, which results in a picture of the rigid ligands' MOs. According to Ref. [31], the effect of the Coulomb field upon the orbital energies within the first-order perturbation theory can be represented as

$$
\left(G^{\Lambda}\right)^{-1}=\left(G_{0}^{\Lambda}\right)^{-1}-\Sigma^{(f)},
$$

where $G_{0}^{\Lambda}$ is the Green's function for the free ligand and the self-energy term $\Sigma^{(f)}$ is due to the external field. The perturbed Green's function $\mathrm{G}^{\boldsymbol{\Lambda}}$ has the same form as before:

$$
\left(G^{\Lambda}(\epsilon)\right)_{L L}^{\mathrm{adv}}=\sum_{i \in L} \lim _{\delta \rightarrow 0^{+}} \frac{\left(c_{i L}^{\Lambda}\right)^{2}}{\epsilon-\epsilon_{i}+i \delta^{\prime}}
$$

but its poles are expressed through the orbital energies of the free ligand $\epsilon_{i}^{(0)}$ and the self-energy parts $\sum_{i i}^{(f)}$ :

$$
\boldsymbol{\epsilon}_{i}=\boldsymbol{\epsilon}_{i}^{(0)}+\sum_{i i}^{(f)} .
$$

The self-energy $\Sigma_{i i}^{(f)}$ is expressed through the partial electron densities by the relation

$$
\begin{aligned}
\sum_{i i}^{(f)} & \approx-\left(Z_{M}-n_{d}\right) e^{2} \sum_{\substack{N \in \Lambda \\
\alpha \in N}} \frac{c_{\alpha i}^{2}}{R_{N}}-e^{2} \sum_{\substack{\Lambda^{\prime} \neq \Lambda \\
N^{\prime} \in \Lambda^{\prime}}} Q_{N^{\prime}} \sum_{\substack{N \neq N^{\prime} \\
\alpha \in N}} \frac{c_{\alpha i}^{2}}{R_{N N^{\prime}}} \\
& =-e^{2} \sum_{N \in \Lambda} c_{\alpha i}^{2}\left(\frac{\left(Z_{M}-n_{d}\right)}{R_{N}}+\sum_{\substack{\Lambda^{\prime} \neq \Lambda \\
N^{\prime} \in \Lambda^{\prime}}} \frac{Q_{N^{\prime}}}{R_{N N^{\prime}}}\right) .
\end{aligned}
$$

These formulae comprise the rigid ligands' MO's (RLMO) model of the electronic structure of the l-system of the TMC.

\section{Implementing LECF for Extending MM to TMC}

\section{TOTAL ENERGY WITHIN THE MMECF MODEL}

The basis for integration of the ECF with the MM is proposed in Ref. [14]. According to Ref. [14], the total energy of a TMC in its $n$th electronic state in the ECF approximation is

$$
\begin{gathered}
E=E_{L}+E_{d}^{\mathrm{eff}}(n) \\
E_{d}^{\mathrm{eff}}(n)=\left\langle\Psi_{n}^{d}\left|H_{d}^{\mathrm{eff}}\right| \Psi_{n}^{d}\right\rangle .
\end{gathered}
$$

The conjecture [17] is that the ligand energy $E_{L}$ can be replaced by $E_{M M}$ - the $M M$ approximate energy. In the present work, we also assume that the effective $d$-shell electron energy $E_{d}^{\text {eff }}(n)$ specific for the $n$th electronic spin state that ultimately comes from the LECF method can be estimated with use of approximations formulated in above:

$$
E=E_{M M}+E_{d}^{\text {eff }}(n) .
$$

In the previous section, we presented derivation for general formulae that comprise the perturbation theory-based approach to evaluation of the Green's function of the l-subsystem using those of the separate free ligands as a zero approximation. Obtained $l$-system Green's function can be estimated for arbitrary molecular geometry without the SCF calculation for the whole $l$-system, computationally more feasible than the SCF procedure itself. Inserting this form of the $l$-system Green's function into the local ECF formulae Eq. (13) yields an estimate for the crystal field acting on the $d$-shell of a central TMI for each molecular configuration of the TMC.

In Eq. (24), the term $E_{d}^{\text {eff }}(n)$ corresponds to the effective $d$-shell energy calculated as the $n$th eigen- 
state of the effective $d$-shell Hamiltonian Eq. (1). The crystal field part of this Hamiltonian is estimated in the framework of the local ECF approach Eq. (13). In the previous sections, we proposed the rigid ligand's MO model that represents the Green's function $G_{0}^{l}$ Eqs. (18) and (19) including only the ligand $\mathrm{MO}$ energy shifts with $\sum_{i i}^{(f)}$ of Eq. (22) calculated with use of the partial charges calculated for free ligands.

\section{PARAMETERS USED IN THE MMGK-LECF APPROACH}

The hybrid scheme involving MM component requires extensive parameterization. The entire set of parameters consists of three subsets. These are the subsets relevant for description of the free $d$ shell, the parameters of the MM part, and those relevant to the junction between the MM and ECF parts.

\section{d-Shell Parameters}

The $d$-shell electronic parameters are taken from the ECF method without changes. They are specific exponents of atomic orbitals, $d$-electron core attraction parameter $U_{d d}$, and the Racah parameters of electron-electron Coulomb repulsion in the $d$-shell $B$ and $C$ specific either for the complex (if known) or their standard values for the free ion tabulated, say, in Ref. [25]. They are described in detail in Ref. [23].

\section{Parameters}

The organic part of a molecule and metal ion coordination sphere, leaving out effects of the $d$ shell, is described in the present hybrid procedure in terms of the MMGK method. According to the MMGK, the arrangement of the donor atoms around the metal is dictated by mutual repulsion between all metal-ligand (M-L) bonds. It is assumed that the repulsion occurs between the effective centers of repulsion lying on the M-L bonds on the distance $r_{\text {eff }}$ from the metal ion. This term implicitly accounts for the electronic effects in the coordination sphere that could not be described within bare ECF formalism (which considers only $d$-electron splitting). Energy of the "bond repulsion" in the coordination sphere is

$$
E_{i j}^{\mathrm{rep}}=A_{i} A_{j} / R_{i j}^{6}
$$

where

$$
\begin{aligned}
R_{i j}^{2} & =r_{i, \mathrm{eff}}^{2}+r_{j, \mathrm{eff}}^{2}-2 r_{i, \mathrm{eff}} r_{j, \mathrm{eff}} \cos \left(X_{i} \hat{M} X_{j}\right) ; \\
r_{i, \mathrm{eff}} & =R\left(M-X_{i}\right) d_{i, \mathrm{eff}} ;
\end{aligned}
$$

$R\left(\mathrm{M}-\mathrm{X}_{i}\right)$ is the actual $\mathrm{M}-\mathrm{X}_{i}$ bond length; $A_{i}, A_{j}$ $d_{\text {eff }, i}, d_{\text {eff }, j}$ are new force-field parameters, characterizing energy of repulsion $(A)$ and positions of the centers of repulsion $\left(d_{\text {eff }}\right)$.

The total MMGK conformation energy of a molecule is

$$
\begin{aligned}
E_{M M}=\sum E_{b}+\sum E_{\text {ang }}+\sum E_{\text {tors }}+ & \sum E_{\mathrm{nb}}+\sum E_{\mathrm{imp}} \\
& +\sum E_{\mathrm{rep} \prime}
\end{aligned}
$$

where the energy terms are

$$
E_{b}=0.5 K_{r}\left(r-r_{0}\right)^{2}
$$

the energy of bond stretching;

$$
E_{b}=D_{0}\left[e^{-\alpha\left(r-r_{0}\right)}-1\right]^{2}
$$

Morse function to allow large variations in metaldonor atom bond lengths in different TMC spin states for:

$$
E_{\text {ang }}=0.5 K_{\theta}\left(\theta-\theta_{0}\right)^{2}
$$

the energy of valence angle bending; note that angles around the metal ion are not considered as they are described through Gillespie-Kepert term.

$$
E_{\text {tors }}=0.5 V_{0}(1+\cos [n(\phi+\psi)])
$$

the energy of torsion interaction;

$$
E_{\mathrm{nb}}=\epsilon_{i j}\left(\frac{r_{0}}{r_{i j}}\right)^{12}-2 \epsilon_{i j}\left(\frac{r_{0}}{r_{i j}}\right)^{6}
$$

the energy of nonbonded interaction;

$$
E_{\mathrm{imp}}=0.5 K_{\mathrm{imp}} \delta^{2}
$$

the energy of improper torsion (out-of-plane) interaction.

MM parameters for organic part of molecule were primarily taken from the CHARMM force field [36, 37], while specific metal-dependent parameters are fitted within the MMECF method. The full set of the MMGK force-field parameters is 
available on request. The same parameters are used for any spin state.

\section{Junction Parameters}

Because within the LECF energy part the entire system is divided into two parts, that is, the $d$-shell and the ligands' $l$-system, their junction requires separate attention. There are two kinds of parameters corresponding, respectively, to the $d-l$ interaction and to the $l$-system electronic structure characterized below.

$d-l$ Interaction Parameters. In the original ECF theory, the specific parameters describing the interaction between the subsystems were fitted to reproduce the $d$-level splitting for octahedral complexes with a specific donor atom. The set of the intersystem interaction parameters includes the $g_{s d}$ and $\bar{g}_{p d}$ parameters of the Coulomb interaction between $d$ shell and transition metal valence $s$ - and $p$-electrons taken from Oleari's work [38], the valence state ionization potentials for the $d$-shell and the donor atoms taken from [39], and the dimensionless factors $\beta_{M L}$ characteristic for a metal-donor atom pair, scaling the resonance interaction. These parameters are transferred from the original ECF [23] to the MMECF package without change.

Electronic Structure Parameters of the l-System. The original ECF [23] method employs the CNDO approximation [40] to estimate the parameters of the electronic structure of the $l$-system required for the calculation of the effective Hamiltonian Eq. (1). These quantities (one-electron densities, orbital energies, and MO-LCAO expansion coefficients) had to be calculated for each molecular geometry by the approximate SCF procedure extended to the entire $l$-system. In the RLMO approximation, these quantities are estimated pertrubatively, more economic from the computational point of view but requiring a larger number of parameters. Within the RLMO model, the electronic structure of the free ligand prototype is supposed to be unchanged during the complex formation. Thus, for the LECF calculations we used the charge distribution calculated for the free ligand itself, that is, the effective point charges are found from the CNDO calculation on the free ligand and considered as electronic structure parameters for the $l$-system. Also, the orbital energies of the ligand MOs having nonzero contribution to the lone pair of the donor atom calculated for the free ligand are to be fed to the MMECF procedure.
They are used to estimate the positions of the poles of the Green's function in the Coulomb field of the charges within the complex according to Eq. (21) with use of the partial densities of the $i$ th MOs on the atom $A$ of the ligand (fragment) $\Lambda$ :

$$
\rho_{i A}=\sum_{\alpha \in A} c_{i \alpha}^{2}
$$

also considered as parameters (here, $c_{i \alpha}$ are $\mathrm{MO}$ LCAO coefficients of the free ligand). Finally, the expansion coefficients of the LP $c_{\alpha L}$ used in Eqs. (11) and (12), where $\alpha$ runs over the donor atom AOs having the dominating contribution to the LP, and calculated within the ligand fixed coordinate frame (LFCF, see below) are treated as parameters of the electronic structure of the $l$-system. All these quantities are calculated with use of the CNDO parameterization for free ligands.

\section{Results and Discussion}

In our present study, the basic procedure for treating PESs of TMCs within the MM approach is constructed. To do so we, first, reformulate in the local form, that is, in terms of the field increments induced by the lone pairs of the ligands, the semiempirical ECF theory (which previously allowed us to calculate with appropriate accuracy the crystal field induced by the ligands on the TMC's $d$-shells). This allowed us to find explicit formulae for the crystal field matrix expressed through the electronic characteristics of the free ligands lone pairs and the procedure to calculate them. In the framework of our approach, the crystal field matrix is calculated for arbitrary arrangement and orientation of the ligands around the central TMI. This puts our approach into a close relation with the empirical angular overlap model (AOM) theory [21, 30]. The latter, despite its purely empirical character, is widely used for interpretation of experimental data on electronic structure of the $d$-shell in TMCs. Because the AOM-based approaches to description of TMIs in the MM framework are present in the literature $[19,20]$, let us discuss briefly the relation and difference between the $\mathrm{AOM}$ and the local ECF.

\section{LOCAL ECF AND THE AOM}

The formulae Eqs. (12) and (13) of the local version of the ECF theory are close to formal expres- 
sions used to substantiate the AOM [21]. As mentioned in the Introduction, the AOM is used as a basis for constructing the CLFSE approach to incorporation of TM into MM [19, 20]. Here, we establish a relation between the $\mathrm{AOM}$ and the local ECF methods.

According to the AOM, the crystal field is given by the sum (cellular expansion [21])

$$
\begin{aligned}
V_{\mu \nu}=\left\langle d_{\mu}\left|V_{c f}\right| d_{\nu}\right\rangle= & \sum_{l} v_{\mu \nu}^{l} \\
& =\sum_{l}\left(v_{\mu \nu}^{l}\right)_{\text {stat }}+\sum_{l}\left(v_{\mu \nu}^{l}\right)_{\mathrm{dyn},}
\end{aligned}
$$

where the sum over $l$ is extended to all the cells [21] that are not clearly defined. According to the AOM, the cellular contributions into the crystal field matrix element $v_{\mu \nu}^{l}$ are related to the cellular AOM parameters $e_{\lambda \lambda^{\prime}}^{l}$ through a purely geometric relation:

$$
\begin{aligned}
v_{\mu \nu}^{l} & =\sum_{\lambda \lambda^{\prime}} R_{\mu \lambda}^{l+} \lambda_{\lambda \lambda^{\prime}}^{l}, R_{\lambda^{\prime} \nu}^{l} \\
\mathbf{v}^{l} & =\mathbf{R}^{l+} \mathbf{e}^{l} \mathbf{R}^{l},
\end{aligned}
$$

where the transformation coefficients $R_{\lambda \mu}^{l}$ form a unitary matrix $\mathbf{R}^{l}$ that transforms $d$-orbitals from the global (laboratory) coordinate frame (GCF) to the diatomic coordinate frame (DCF) related to the ligand $l$. The latter is chosen in such a way that its $z$-axis is the straight line connecting the metal atom with the ligand donor atom. The matrix elements $e_{\lambda \lambda^{\prime}}^{l}$ of the $\mathbf{e}^{l}$ matrix in the DCF are labeled by the indici $\lambda \lambda^{\prime}$ taking values $\sigma, \pi_{x}, \pi_{y}, \delta_{x y}, \delta_{x^{2}-y^{2}}$ according to the symmetry of the metal $d$-orbitals with respect to the $z$-axis of the DCF $[21,30]$. To establish the relation with the local ECF, we naturally identify the Coulomb term of the ECF method Eq. (4) with the static contribution of the AOM and the covalent contribution $W_{\mu \nu}^{\text {cov }}$ of the local ECF method in the form given by Eq. (13) with the dynamic contribution of the AOM. Under these assumptions we can rewrite Eq. (13) as

$$
W_{\mu \nu}^{\mathrm{cov}}=\sum_{\Lambda}\left(v_{\mu \nu}^{\Lambda}\right)_{\mathrm{dyn}},
$$

with

$$
\left(v_{\mu \nu}^{\Lambda}\right)_{\mathrm{dyn}}=\sum_{L \in \Lambda} \beta_{\mu L} \beta_{\nu L} G_{L L}^{\mathrm{adv}}\left(A_{d}\right)
$$

and analogously for the static contribution from Eq. (4)

$$
W_{\mu \nu}^{\text {field }}=\sum_{\Lambda}\left(v_{\mu \nu}^{\Lambda}\right)_{\text {stat }}=\sum_{\Lambda} \sum_{A \in \Lambda} Q_{A} V_{\mu \nu \prime}^{A}
$$

where sum is over all atoms $A$ of the TMC. In Ref. [22], the covalent contribution to the parameters $e_{\lambda \lambda^{\prime}}^{\Lambda}$ was found:

$$
\left(e_{\lambda \lambda^{\prime}}^{\Lambda}\right)_{\mathrm{dyn}}=\sum_{\mu \nu} R_{\mu \lambda}^{\Lambda}\left[\sum_{L \in \Lambda} \beta_{\mu L} G_{L L}^{\mathrm{adv}}\left(A_{d}\right) \beta_{\nu L}\right] R_{\nu \lambda^{\prime},}^{\Lambda}
$$

where $\beta_{\mu L}^{\Lambda}$ is the resonance integral between the $L$ th LMO of the ligand $\Lambda$ and the metal $d$-AOs in the GCF and $R_{\mu \nu}^{\Lambda}$ is the element of orthogonal rotation matrix transforming the GCF to the DCF. Introducing the components of the $t^{L}$ vector of the resonance integrals between the metal $d$-AOs and the $L$ th $\mathrm{LMO}$ in the DCF, we get

$$
t_{\lambda}^{L}=\sum_{\mu} R_{\mu \lambda} \beta_{L \mu} .
$$

and Eq. (32) acquires the form

$$
e_{\lambda \lambda^{\prime}}^{\Lambda}=\sum_{L \in \Lambda} t_{\lambda}^{L} G_{L L}^{\mathrm{adv}}\left(A_{d}\right) t_{\lambda^{\prime}}^{L+} .
$$

Thus

$$
W_{\mu \nu}^{\mathrm{cov}}=\sum_{\Lambda \lambda \lambda^{\prime}} R_{\mu \lambda}^{\Lambda} e_{\lambda \lambda^{\prime}}^{\Lambda} R_{\nu \lambda^{\prime}}^{\Lambda}
$$

The expression (34) defines the AOM parameters in terms of the quantities that can be calculated within the LECF method. The AOM parameter matrices $\mathbf{e}^{\Lambda}$ are controlled by the resonance integrals vector $\mathbf{t}^{L}$, $L \in \Lambda$ in the DCF and by the respective Green's function $G_{L L}^{\text {adv }}\left(A_{d}\right)$. In Ref. [22], Eqs. (32) and (34) have been used to calculate the values of the $e_{\sigma}$ and $e_{\pi}$ parameters for a series of octahedral complexes with nitrogen-containing ligands. That calculation was in good agreement with experimental $10 D q$ values (within $10 \%$ accuracy).

The above explicit formulae for the $e_{\lambda \lambda^{\prime}}^{\Lambda}$ parameters allow us to perform analysis of various approximations employed in the literature. For example, in Ref. [19] the dependence of the AOM parameters $e_{\sigma}$ on separation between the ligand donor and metal atoms is parameterized in the form 


$$
e_{\sigma}=e_{\sigma}^{0}+e_{\sigma}^{\prime}\left(r-r_{0}\right) .
$$

Even the original AOM method is somewhat richer than it is parameterized in the CLFSE model $[19,20]$ because it presumes existence of off-diagonal parameters $e_{\lambda \lambda^{\prime}}^{\lambda}$ for misdirected lone pairs. These quantities are not parameterized in the CLFSE scheme. Analysis of Eqs. (32)-(34) reveals that the AOM parameters should not possess the above simple linear form. By contrast, they depend on the orientation of the ligand. This dependence can be analyzed as follows: Let us consider the vector $\tilde{\mathbf{c}}_{L}$, having the components $\tilde{c}_{L s}, \tilde{c}_{L x}, \tilde{c}_{L y}$, and $\tilde{c}_{L z}$ in the $D C F$, representing the LP expansion over the donor atom AOs (here we assume that each donor atom gives one $s$ - and three $p$-AO to the complex $\mathrm{AO}$ basis). The resonance integrals $t_{\lambda}^{L}$ can be easily determined through the resonance integrals between the metal $d-\mathrm{AO}$ and the donor atom $\mathrm{AOs} \beta_{\mu \alpha}$ within the DCF $\left(\mu=z^{2}, x^{2}-y^{2}, x z, x y, y z ; \alpha=s, x, y, z\right)$ :

$$
\begin{aligned}
t_{\sigma}^{L} & =\tilde{c}_{L s} \beta_{z^{2}, s}+\tilde{c}_{L z} \beta_{z^{2}, p_{z}} \\
t_{\pi x}^{L} & =\tilde{c}_{L x} \beta_{x z, x} \\
t_{\pi y}^{L} & =\tilde{c}_{L y} \beta_{y z, y} .
\end{aligned}
$$

Components of the $\delta$-symmetry do not appear unless $\mathrm{d}$-orbitals are represented among the AOs. The $\beta_{\mu \alpha}$ components depend only on the interatomic separation. The LMO-AO coefficients for $p$-AOs in the DCF can be expressed through the invariant three-component vector $\overline{\mathbf{c}}_{L p}$ of the $p$-AOs coefficients in the LPs expansion calculated for some LFCF, which are the parameters of the $l$-system electronic structure (see above). The LFCF coincides neither with the GCF nor with the DCF being rigidly attached to the ligand as, for instance, the frame of the principal axes of the inertia tensor of the isolated ligand is. In this article, we assume that a rule for choosing the LFCF in terms of the donor atom and its neighbor atoms is somehow established. Then, the relation between the $\tilde{\mathbf{c}}_{L p}$ and the $\overline{\mathbf{c}}_{L p}$ parameters has the form

$$
\tilde{\mathbf{c}}_{L p}=\mathcal{T}^{\Lambda} \overline{\mathbf{c}}_{L p}
$$

where $\mathcal{T}^{\Lambda}$ is the rotation matrix transforming the LFCF related to the ligand $\Lambda$ to the DCF. (For any coordinate frame $\tilde{c}_{L S}=\bar{c}_{L S}$ because the coefficient of the $s$-function in the LP expansion is rotation-invariant parameter of the $l$-system electronic struc- ture). Eq. (38) thus expresses the dependence of the $\mathrm{AOM}$ parameters and thus of the crystal field on molecular geometry of the TMC in a more precise manner than is done in the CLFSE formalism.

\section{IMPLEMENTATION}

The main goal was to construct a procedure comprising the LECF approach and general MM by Eq. (24). It is implemented in the MMECF package [41] with the RLMO version of the electronic structure of the ligand system. The package includes both gradient minimization and minimization without derivatives $[42,43]$ procedures. The package allows us to consider ligands or its fragments as rigid bodies. As a consequence, the number of geometry variables considerably decreases, which speeds the minimization procedure. Technically, the rigid bodies first preoptimized with use of the MM potentials only (excluding metal-dependent ones) and in further calculations their internal geometry is fixed.

\section{SPIN STATES AND GEOMETRIES OF IRON (II) COMPLEXES}

A series of complexes of $\mathrm{Fe}^{2+}$ were chosen to check the relevance of the proposed scheme and parameterize it. This set contains complexes of both mono- and polydentate ligands. We studied the $\left[\mathrm{Fe}(\mathrm{Py})_{6}\right]^{2+},\left[\mathrm{Fe}(\mathrm{BiPy})_{3}\right]^{2+},\left[\mathrm{Fe}(\mathrm{TerPy})_{2}\right]^{2+}$, and $\left[\mathrm{Fe}(\mathrm{mBiPy})_{3}\right]^{2+}$ complexes (the ligands are shown in Fig. 1). Both the low- and high-spin complexes are present in the list. In the case of the RLMO model, however, the $d$-shell splitting is too strong as compared to experiment so we limit ourselves to optimal geometry calculation for different spin states.

Initial geometries were taken from the Cambridge Crystal Structure Data Bank (CCSDB). Hydrogen atoms were added where necessary. Corresponding CCSDB code names of the complexes are PYFEFE [44], VEWVEY [45], NUZKOI [46], and ZIMBUS [47]. We first preoptimized geometry of the ligands using the pure MM procedure and treated the six-membered aromatic rings as rigid fragments (having only six degrees of freedom each). Minimization without derivatives was used with convergence criterion (energy change per iteration $10^{-6} \mathrm{kcal} / \mathrm{mol}$ ).

Because the original ECF method was shown previously [23] to provide an accurate description for the crystal field in TMC's itself and also for its dependence on tiny geometry variations [35, 48], 


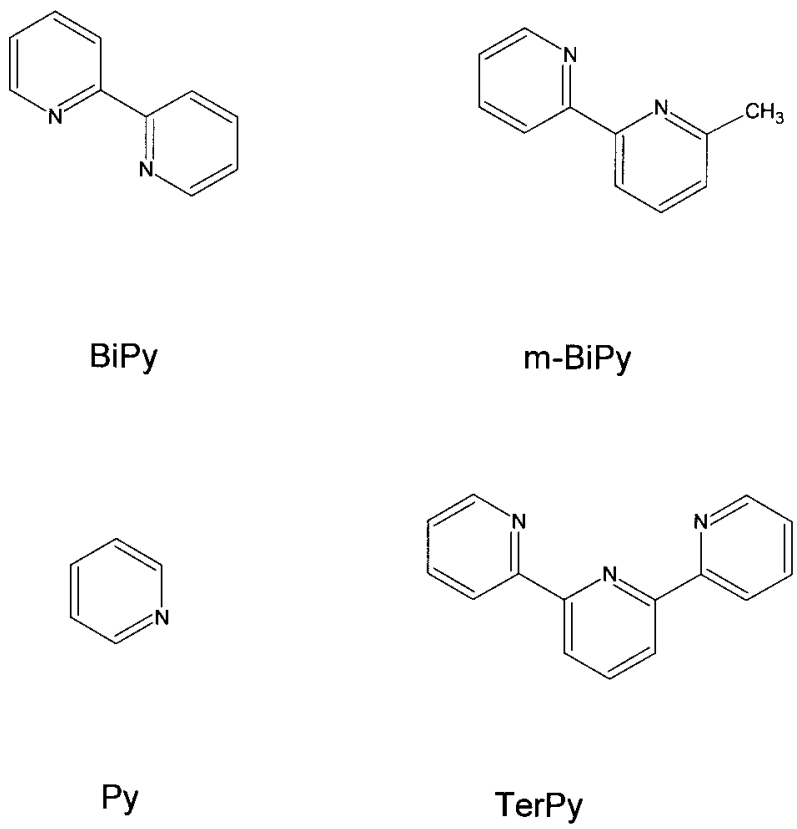

FIGURE 1. Ligands used in the calculations.

we first fitted the MM parameters describing the $\mathrm{Fe}-\mathrm{N}$ interactions (because, namely, this part of the MM model is affected by introducing the LECF procedure for estimating the $d$-shell energy). The parameters fit was performed by trial-and-error with the condition that the total spin for the global energy minimum should coincide with the experiment and the geometry structure at this minimum should be close to the experimental one. Within such a setting we, first, studied the complexes listed above with use of the standard values $\left(B_{0}=917\right.$ $\mathrm{cm}^{-1}, C_{0}=4040 \mathrm{~cm}^{-1}$ ) of the Racah parameters for the free $\mathrm{Fe}^{2+}$ ion [25].

This yielded the results present in Table I column $\eta=1$ (see below). The general conclusion drawn is that the general outline of the potential energy profiles is in agreement with experimental data, although the important details are still wrong. Indeed, with the free ion values of the Racah parameters the high-spin molecules have the total spin 2 in their deepest minima and the low-spin ones the total spin 0 , in agreement with experiment [16]. However, the energy differences between the minima corresponding to the correct total spin and other values of the total spin are too small (ca. 1 $\mathrm{kcal} / \mathrm{mol}$ ) for all molecules considered. On the other hand, the geometry for the high-spin complexes is systematically shifted toward longer $\mathrm{Fe}-\mathrm{N}$ separations. In fact, it looked like the intersection of the high- and low-spin terms in the com- plexes of iron(II) took place in our calculation at about $2.2 \AA$ rather than at $2.1 \AA$ as known to be from the experiment and previous ECF calculation [14]. To clarify this situation, we calculated the $10 D q$ splitting parameter as a function of the metal-donor atom distance both with the use of the LECF combined with the rigid ligand MOs model (LECFRLMO) and by the original ECF method for the octahedral configuration of the complex $\left[\mathrm{Fe}(\mathrm{Py})_{6}\right]^{2+}$. The result is shown in Figure 2. It can be characterized in a twofold manner: First, one can notice that the LECF-RLMO curve can be approximately obtained from the exact ECF one by $0.2 \AA$ shift to the larger interatomic separations; second, it can be checked that for the sensitive range of the interatomic separations about $2 \AA$ the ratio $10 D q($ LECF-RLMO) $/ 10 D q(\mathrm{ECF})$ falls in the interval 1.5-2.2.

These observations can be analyzed with use of the Tanabe-Sugano diagrams (see, e.g., [25]). Indeed, as one remembers, the relative position of the electronic terms of different total spin depends on the $10 \mathrm{Dq} / \mathrm{B}$ ratio, with its large values corresponding to the high-spin ground states. Because the LECF-RLMO model regularly overestimates the splitting parameter due to neglect of the one-electron transfers between the ligands and the metal 4sand $4 p$-AOs the correct position of the cross-section point of the high- and low-spin terms can be obtained by multiplying the free ion $B_{0}$ and $C_{0}$ values by a factor $\eta$. We studied the complex geometries for four values of the $\eta$ parameters equal respectively to $1,1.5,2$, and 2.4 . For each value of $\eta$, the $\mathrm{MM}$ parameters for the $\mathrm{Fe}-\mathrm{N}$ interaction were fitted. Obtained calculation results are summarized in Tables I and II. The calculation details characterizing the quality of the results for corresponding four series of the Racah $B$ and $C$ parameters are presented in Table I, while in Table II the information for the best-fit geometries with the Racah $B$ and $C$ parameters equal to 1430 and $6240 \mathrm{~cm}^{-1}$, respectively (which corresponds to $\eta=1.5$ ), is presented.

The nearly octahedral $\left[\mathrm{Fe}(\mathrm{Py})_{6}\right]^{2+}$ complex has high-spin ground state. Our calculations reproduce the same spin of the ground state. The coordination bond lengths are in acceptable agreement with experimental data. Valence angles at the metal are in good agreement with experiment as well. However, the torsional angles between pyridine planes differ significantly from the experimental ones. This may be explained by nonbonded interactions in crystal environment. Electronic terms for $\left[\mathrm{Fe}(\mathrm{Py})_{6}\right]^{2+} \mathrm{Com}-$ plex for different $\eta$ values are presented in Figure 3. 
TABLE I

Calculation details for six-coordinated iron(II) complexes.

\begin{tabular}{|c|c|c|c|c|c|c|c|c|c|}
\hline \multirow[b]{3}{*}{ Complex } & \multirow[b]{3}{*}{ Spin } & \multicolumn{4}{|c|}{$\eta 1$} & \multicolumn{4}{|c|}{$\eta 1.5$} \\
\hline & & \multirow[b]{2}{*}{$E(\mathrm{kcal} / \mathrm{mol})$} & \multicolumn{3}{|c|}{ RMS } & \multirow[b]{2}{*}{$E(\mathrm{kcal} / \mathrm{mol})$} & \multicolumn{3}{|c|}{ RMS } \\
\hline & & & Bonds & Angles & Tors. & & Bonds & Angles & Tors. \\
\hline \multirow{6}{*}{$\mathrm{Fe}(\text { bipy })_{3}^{2+}$} & 0 & -9367.42 & 0.03 & 1.2 & 1.2 & -9336.11 & 0.01 & 1.4 & 0.8 \\
\hline & & & 0.08 & 0.7 & & & 0.00 & 1.9 & \\
\hline & 1 & -9340.31 & 0.09 & 2.3 & 1.6 & -9297.27 & 0.06 & 1.7 & 1.3 \\
\hline & & & 0.24 & 3.7 & & & 0.16 & 2.3 & \\
\hline & 2 & -9354.38 & 0.12 & 4.2 & 5.0 & -9322.18 & 0.08 & 2.9 & 3.4 \\
\hline & & & 0.32 & 8.9 & & & 0.23 & 6.2 & \\
\hline \multirow[t]{6}{*}{$\mathrm{Fe}(\text { terpy })_{2}^{2+}$} & 0 & -9337.06 & 0.05 & 1.5 & 0.8 & -9301.69 & 0.02 & 1.5 & 0.9 \\
\hline & & & 0.12 & 0.6 & & & 0.03 & 1.9 & \\
\hline & 1 & -9315.77 & 0.10 & 2.8 & 1.9 & -9269.24 & 0.07 & 2.1 & 2.1 \\
\hline & & & 0.26 & 2.9 & & & 0.18 & 1.2 & \\
\hline & 2 & -9326.85 & 0.14 & 5.3 & 3.6 & -9288.03 & 0.11 & 4.4 & 3.4 \\
\hline & & & 0.39 & 11.3 & & & 0.30 & 9.4 & \\
\hline \multirow[t]{6}{*}{$\mathrm{Fe}(\mathrm{py})_{6}^{2+}$} & 0 & -9382.08 & 0.06 & 0.8 & 16.3 & -9336.25 & 0.09 & 0.7 & 21.5 \\
\hline & & & 0.15 & 1.4 & & & 0.23 & 1.2 & \\
\hline & 1 & -9365.24 & 0.05 & 0.7 & 30.5 & -9316.22 & 0.07 & 0.7 & 24.6 \\
\hline & & & 0.13 & 1.1 & & & 0.18 & 1.2 & \\
\hline & 2 & -9382.49 & 0.02 & 0.7 & 28.8 & -9345.72 & 0.02 & 0.7 & 24.9 \\
\hline & & & 0.05 & 1.2 & & & 0.06 & 1.2 & \\
\hline \multirow[t]{7}{*}{$\mathrm{Fe}(\mathrm{m}-\mathrm{bipy})_{3}^{2+}$} & 0 & -9356.69 & 0.04 & 4.3 & 4.4 & -9313.41 & 0.07 & 4.9 & 5.1 \\
\hline & & & 0.12 & 8.9 & & & 0.20 & 9.9 & \\
\hline & 1 & -9337.31 & 0.03 & 3.0 & 4.1 & -9289.08 & 0.04 & 3.3 & 4.7 \\
\hline & & & 0.08 & 6.1 & & & 0.12 & 6.6 & \\
\hline & 2 & -9358.23 & 0.04 & 1.7 & 2.5 & -9324.60 & 0.02 & 1.6 & 2.5 \\
\hline & & & 0.10 & 3.7 & & & 0.03 & 3.3 & \\
\hline & & \multicolumn{4}{|c|}{$\eta 2$} & \multicolumn{4}{|c|}{$\eta 2.4$} \\
\hline \multirow[t]{6}{*}{$\mathrm{Fe}(\mathrm{bipy})_{3}^{2+}$} & 0 & -9235.59 & 0.01 & 1.7 & 1.3 & -9209.11 & 0.02 & 1.9 & 1.2 \\
\hline & & & 0.02 & 2.4 & & & 0.05 & 3.1 & \\
\hline & 1 & -9197.34 & 0.04 & 1.6 & 0.8 & -9165.73 & 0.03 & 1.5 & 1.0 \\
\hline & & & 0.11 & 1.3 & & & 0.08 & 1.6 & \\
\hline & 2 & -9233.85 & 0.06 & 1.9 & 2.3 & -9209.02 & 0.04 & 1.5 & 1.8 \\
\hline & & & 0.16 & 3.8 & & & 0.11 & 2.7 & \\
\hline \multirow{6}{*}{$\mathrm{Fe}(\text { terpy })_{2}^{2+}$} & 0 & -9198.44 & 0.01 & 1.6 & 1.3 & -9172.85 & 0.02 & 1.8 & 1.2 \\
\hline & & & 0.01 & 2.2 & & & 0.03 & 3.0 & \\
\hline & 1 & -9163.55 & 0.05 & 1.9 & 1.3 & -9127.79 & 0.04 & 1.7 & 1.8 \\
\hline & & & 0.14 & 0.7 & & & 0.10 & 1.4 & \\
\hline & 2 & -9187.09 & 0.08 & 3.2 & 2.3 & -9156.94 & 0.06 & 2.7 & 2.1 \\
\hline & & & 0.23 & 6.8 & & & 0.18 & 5.7 & \\
\hline \multirow{6}{*}{$\mathrm{Fe}(\mathrm{py})_{6}^{2+}$} & 0 & -9233.06 & 0.10 & 0.7 & 21.2 & -9197.95 & 0.11 & 0.7 & 21.0 \\
\hline & & & 0.26 & 1.1 & & & 0.30 & 1.1 & \\
\hline & 1 & -9212.45 & 0.08 & 0.7 & 20.3 & -9173.28 & 0.09 & 0.7 & 20.5 \\
\hline & & & 0.21 & 1.1 & & & 0.24 & 1.1 & \\
\hline & 2 & -9257.70 & 0.04 & 0.7 & 23.6 & -9227.56 & 0.06 & 0.7 & 22.8 \\
\hline & & & 0.11 & 1.2 & & & 0.16 & 1.1 & \\
\hline \multirow{6}{*}{$\mathrm{Fe}(\mathrm{m}-\mathrm{bipy})_{3}^{2+}$} & 0 & -9208.88 & 0.08 & 5.4 & 5.6 & -9177.23 & 0.09 & 5.7 & 6.4 \\
\hline & & & 0.22 & 10.7 & & & 0.26 & 11.1 & \\
\hline & 1 & -9192.56 & 0.06 & 4.5 & 6.1 & -9156.17 & 0.07 & 4.7 & 6.4 \\
\hline & & & 0.18 & 9.1 & & & 0.21 & 9.4 & \\
\hline & 2 & -9231.67 & 0.03 & 2.4 & 3.1 & -9204.35 & 0.04 & 2.7 & 4.1 \\
\hline & & & 0.07 & 4.8 & & & 0.12 & 5.2 & \\
\hline
\end{tabular}




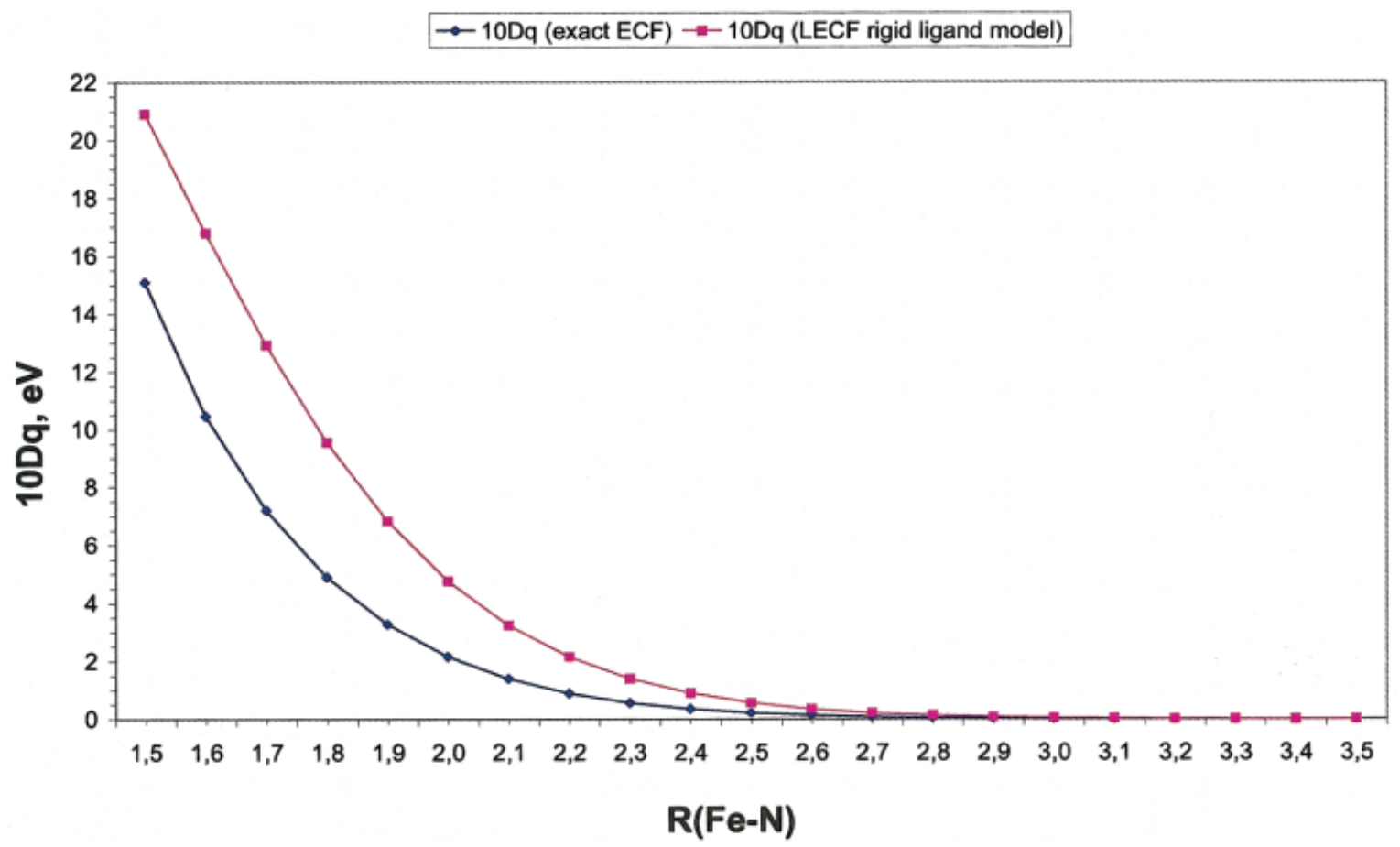

FIGURE 2. Dependence of $10 D q$ for calculations by ECF or LECF-RLMO on Fe- $\mathrm{N}$ separation. [Color figure can be viewed in the online issue, which is available at www.interscience.wiley.com.]

They illustrate the validity of our conjecture concerning the position of the spin-cross-section point as a function of $\eta$. As one can see for stronger Coulomb interaction in the $d$-shell the transition to the low-spin ground state requires stronger crystal filed. This results in the shift of the spin-crosssection point to the experimentally substantiated value of $2.1 \AA$.

Tris-(2-methyl-bipyridine)-iron complex $\left[\mathrm{Fe}(\mathrm{mBiPy})_{3}\right]^{2+}$ also has the high-spin ground state, in agreement with the experiment. The calculated lengths of the $\mathrm{Fe}-\mathrm{N}$ bonds for the methylated ring are somewhat shorter than the experimental ones. This disagreement can possibly be cured by adjusting the 1,4-van der Waals parameters, which were not fitted in the present study.

Geometry of both low-spin complexes, $\left[\mathrm{Fe}(\mathrm{BiPy})_{3}\right]^{2+}$ and $\left[\mathrm{Fe}(\mathrm{TerPy})_{2}\right]^{2+}$, are calculated with good accuracy. Even the details of geometry in the case of the terpyridyl complex are reproduced: The bond length for the central ring is somewhat longer than that for the side rings. Despite the difference in bond distances, one can see a good quality of experimental geometry reproduction by the LECFRLMO-MMGK calculations. Moreover, the hybrid method predict the minimum energy for each complex to the spin state observed experimentally. The energy differences between the minima for the bestfit Racah parameters are large enough to explain the fact that for the compounds under consideration only one ground spin state is observed.

Finally, we can say that by concert usage of the LECF-RLMO procedure as a QM model for describing the geometry dependence of the $d$-shell energy and the MMGK procedure as a MM model for the ligand energy, a unified MM-like description for the PESs of different spin states of the iron(II) complexes is achieved with use of the single spin-independent parameterization (the full set of parameters are available from the authors upon request).

A similar result has been reached in Ref. [19] for a series of nickel (II) complexes with use of the CLFSE-MM methodology. However, the description of the $d$-shell state in the CLFSE-MM method [19] is based on the one-electron approximation and neglects the $d$-electron correlations-which, in fact, are responsible for the form of the energy lowexcitation spectrum of the $d$-shell. For that reason, the success of the CLFSE method in describing molecules with different total spin is reached for the case of nickel (II) complexes when the one-electron approximation suffices to describe the difference between the $d$-level splitting and filling for the 
TABLE II

Observed and calculated bond lengths (Å) and angles $\left(^{\circ}\right)$ for six-coordinated iron(II) complexes.

\begin{tabular}{|c|c|c|c|c|c|c|c|}
\hline \multirow[b]{3}{*}{$\eta$} & \multirow[b]{3}{*}{ Exp. } & \multicolumn{6}{|c|}{ Calc. } \\
\hline & & \multicolumn{2}{|c|}{$S=2$} & \multicolumn{2}{|c|}{$S=0$} & \multicolumn{2}{|c|}{$S=1$} \\
\hline & & 1 & 1.5 & 1 & 1.5 & 1 & 1.5 \\
\hline \multicolumn{8}{|c|}{$\begin{array}{c}{\left[\mathrm{Fe}(\text { bipy })_{3}\right]^{2+} ; \text { ground state }} \\
S=0\end{array}$} \\
\hline Energy (kcal/mol) & & -9354.38 & -9209.02 & -9367.42 & -9209.11 & -9340.31 & -9165.73 \\
\hline \multirow[t]{6}{*}{$\mathrm{Fe}-\mathrm{N}(\AA)$} & 1.97 & 2.29 & 2.19 & 2.05 & 1.97 & 2.24 & 1.94 \\
\hline & 1.97 & 2.29 & 2.19 & 2.05 & 1.97 & 2.00 & 2.15 \\
\hline & 1.97 & 2.29 & 2.19 & 2.04 & 1.97 & 2.00 & 2.17 \\
\hline & 1.97 & 2.29 & 2.19 & 2.04 & 1.97 & 2.24 & 2.17 \\
\hline & 1.97 & 2.29 & 2.20 & 2.05 & 1.96 & 2.27 & 2.15 \\
\hline & 1.97 & 2.29 & 2.20 & 2.04 & 1.96 & 2.27 & 1.93 \\
\hline \multirow[t]{15}{*}{$\mathrm{N}-\mathrm{Fe}-\mathrm{N}\left({ }^{\circ}\right)$} & 81.9 & 73.0 & 75.7 & 82.3 & 84.7 & 79.4 & 82.0 \\
\hline & 94.3 & 94.5 & 94.7 & 93.5 & 93.1 & 97.2 & 94.2 \\
\hline & 89.8 & 100.3 & 98.6 & 90.5 & 88.9 & 93.7 & 87.9 \\
\hline & 94.3 & 94.7 & 94.3 & 94.5 & 93.4 & 97.9 & 96.1 \\
\hline & 174.6 & 161.7 & 166.2 & 175.6 & 177.1 & 169.9 & 177.1 \\
\hline & 174.6 & 159.7 & 164.9 & 174.1 & 176.3 & 175.3 & 172.2 \\
\hline & 94.3 & 93.2 & 94.0 & 93.2 & 92.3 & 97.5 & 96.6 \\
\hline & 89.8 & 102.3 & 98.2 & 90.7 & 89.9 & 89.5 & 92.2 \\
\hline & 94.3 & 95.9 & 96.0 & 94.6 & 92.9 & 94.9 & 96.0 \\
\hline & 81.9 & 73.0 & 75.8 & 82.5 & 84.6 & 79.4 & 76.3 \\
\hline & 94.3 & 94.6 & 94.0 & 93.9 & 93.2 & 94.3 & 95.1 \\
\hline & 89.8 & 99.9 & 95.5 & 89.7 & 89.4 & 89.0 & 88.02 \\
\hline & 174.6 & 161.1 & 164.1 & 174.0 & 176.9 & 167.4 & 170.8 \\
\hline & 94.3 & 94.7 & 93.0 & 92.9 & 92.9 & 95.3 & 94.4 \\
\hline & 81.9 & 73.1 & 75.7 & 82.3 & 84.9 & 73.6 & 82.0 \\
\hline \multicolumn{8}{|c|}{$\begin{array}{c}\left.[\text { Fe(terpy) }]_{2}\right]^{2+} ; \text { ground state } \\
S=0\end{array}$} \\
\hline Energy (kcal/mol) & & -9315.77 & -9156.94 & -9337.07 & -9172.85 & -9326.85 & -9127.79 \\
\hline \multirow[t]{6}{*}{$\mathrm{Fe}-\mathrm{N}(\AA)$} & 1.88 & 2.32 & 2.23 & 1.92 & 1.86 & 1.96 & 1.90 \\
\hline & 1.97 & 2.33 & 2.23 & 2.11 & 2.01 & 2.27 & 2.18 \\
\hline & 1.98 & 2.35 & 2.25 & 2.12 & 2.01 & 2.29 & 2.20 \\
\hline & 1.88 & 2.32 & 2.23 & 1.92 & 1.87 & 1.97 & 1.90 \\
\hline & 1.98 & 2.35 & 2.25 & 2.14 & 2.02 & 2.31 & 2.21 \\
\hline & 1.98 & 2.33 & 2.23 & 2.13 & 2.02 & 2.29 & 2.20 \\
\hline \multirow[t]{15}{*}{$\mathrm{N}-\mathrm{Fe}-\mathrm{N}\left({ }^{\circ}\right)$} & 81.7 & 70.9 & 72.8 & 81.2 & 82.9 & 79.2 & 81.1 \\
\hline & 81.1 & 70.4 & 72.3 & 81.2 & 83.1 & 78.5 & 80.3 \\
\hline & 179.4 & 178.1 & 175.3 & 180.0 & 179.9 & 179.8 & 179.7 \\
\hline & 98.3 & 107.7 & 111.8 & 99.4 & 97.3 & 101.9 & 100.0 \\
\hline & 99.9 & 111.3 & 103.2 & 99.3 & 97.4 & 101.5 & 99.4 \\
\hline & 162.8 & 141.3 & 145.0 & 162.4 & 166.0 & 157.7 & 161.4 \\
\hline & 98.7 & 108.7 & 109.3 & 98.8 & 97.2 & 100.6 & 98.7 \\
\hline & 90.0 & 98.0 & 95.5 & 91.3 & 90.8 & 93.9 & 92.8 \\
\hline & 91.6 & 94.5 & 95.2 & 91.4 & 90.7 & 91.3 & 91.6 \\
\hline & 98.5 & 110.0 & 105.7 & 98.8 & 96.8 & 101.7 & 99.9 \\
\hline & 92.3 & 93.9 & 95.5 & 91.5 & 91.0 & 91.2 & 90.9 \\
\hline & 91.6 & 99.1 & 94.6 & 91.6 & 91.1 & 92.7 & 91.0 \\
\hline & 81.3 & 70.4 & 72.4 & 80.6 & 82.7 & 78.3 & 80.3 \\
\hline & 80.5 & 70.6 & 72.6 & 80.7 & 82.6 & 78.3 & 80.4 \\
\hline & 161.8 & 141.0 & $\begin{array}{c}145.0 \\
\text { (Continued) }\end{array}$ & 161.4 & 165.4 & 156.6 & 160.6 \\
\hline
\end{tabular}


TABLE II

(Continued)

\begin{tabular}{|c|c|c|c|c|c|c|c|}
\hline \multirow[b]{3}{*}{$\eta$} & \multirow[b]{3}{*}{ Exp. } & \multicolumn{6}{|c|}{ Calc. } \\
\hline & & \multicolumn{2}{|c|}{$S=2$} & \multicolumn{2}{|c|}{$S=1$} & \multicolumn{2}{|c|}{$S=0$} \\
\hline & & 1 & 1.5 & 1 & 1.5 & 1 & 1.5 \\
\hline \multicolumn{8}{|c|}{$\begin{array}{l}{\left[\mathrm{Fe}(\mathrm{py})_{6}\right]^{2+} ; \text { ground state }} \\
\quad S=2\end{array}$} \\
\hline Energy (kcal/mol) & & -9382.49 & -9227.56 & -9365.24 & -9173.28 & -9382.08 & -9197.95 \\
\hline \multirow[t]{6}{*}{$\mathrm{Fe}-\mathrm{N}(\AA)$} & 2.25 & 2.30 & 2.20 & 2.29 & 1.95 & 2.09 & 2.00 \\
\hline & 2.28 & 2.30 & 2.21 & 2.04 & 2.20 & 2.14 & 2.05 \\
\hline & 2.29 & 2.30 & 2.20 & 2.29 & 2.19 & 2.11 & 2.02 \\
\hline & 2.26 & 2.30 & 2.20 & 2.29 & 1.95 & 2.09 & 2.01 \\
\hline & 2.22 & 2.30 & 2.21 & 2.04 & 2.20 & 2.13 & 2.05 \\
\hline & 2.25 & 2.30 & 2.20 & 2.29 & 2.19 & 2.10 & 2.02 \\
\hline \multirow[t]{15}{*}{$\mathrm{N}-\mathrm{Fe}-\mathrm{N}\left({ }^{\circ}\right)$} & 90.1 & 90.2 & 90.2 & 90.0 & 90.0 & 90.1 & 90.1 \\
\hline & 89.4 & 89.0 & 89.9 & 87.8 & 90.0 & 90.0 & 90.0 \\
\hline & 178.5 & 179.0 & 179.9 & 179.8 & 180.0 & 180.0 & 180.0 \\
\hline & 90.0 & 90.4 & 90.1 & 90.0 & 90.1 & 90.0 & 90.0 \\
\hline & 90.1 & 90.2 & 89.9 & 92.0 & 90.0 & 89.9 & 89.9 \\
\hline & 90.2 & 90.8 & 90.2 & 90.3 & 90.6 & 90.0 & 90.0 \\
\hline & 89.6 & 89.6 & 89.9 & 89.9 & 89.9 & 90.0 & 89.9 \\
\hline & 178.5 & 178.0 & 179.5 & 179.9 & 179.3 & 179.7 & 179.7 \\
\hline & 89.6 & 89.3 & 90.1 & 90.1 & 90.0 & 90.2 & 90.0 \\
\hline & 92.1 & 90.0 & 90.0 & 92.1 & 90.2 & 90.1 & 90.0 \\
\hline & 88.3 & 91.2 & 90.3 & 89.8 & 90.2 & 90.3 & 90.2 \\
\hline & 179.4 & 179.2 & 179.6 & 179.6 & 179.4 & 179.8 & 179.8 \\
\hline & 90.3 & 89.9 & 89.9 & 90.1 & 90.0 & 89.9 & 90.0 \\
\hline & 88.5 & 90.8 & 90.2 & 88.1 & 90.1 & 90.1 & 90.2 \\
\hline & 91.9 & 88.7 & 89.4 & 89.8 & 89.3 & 89.5 & 89.8 \\
\hline \multicolumn{8}{|c|}{$\begin{array}{c}{\left[\mathrm{Fe}(\mathrm{m}-\mathrm{bipy})_{3}\right]^{2+} ; \text { ground state }} \\
\quad S=2\end{array}$} \\
\hline Energy (kcal/mol) & & -9358.23 & -9204.35 & -9337.31 & -9156.17 & -9356.69 & -9177.23 \\
\hline \multirow[t]{6}{*}{$\mathrm{Fe}-\mathrm{N}(\AA)$} & 2.20 & 2.29 & 2.20 & 2.21 & 2.12 & 2.04 & 1.96 \\
\hline & 2.16 & 2.30 & 2.20 & 2.02 & 1.95 & 2.04 & 1.97 \\
\hline & 2.20 & 2.30 & 2.21 & 2.28 & 2.18 & 2.02 & 1.96 \\
\hline & 2.19 & 2.31 & 2.21 & 2.11 & 2.01 & 2.16 & 2.06 \\
\hline & 2.26 & 2.30 & 2.21 & 2.29 & 2.20 & 2.14 & 2.05 \\
\hline & 2.24 & 2.31 & 2.21 & 2.30 & 2.20 & 2.16 & 2.07 \\
\hline \multirow[t]{15}{*}{$\mathrm{N}-\mathrm{Fe}-\mathrm{N}\left({ }^{\circ}\right)$} & 88.3 & 93.6 & 92.8 & 93.1 & 90.9 & 91.2 & 90.1 \\
\hline & 170.1 & 177.2 & 173.9 & 176.0 & 171.2 & 178.3 & 175.6 \\
\hline & 74.5 & 73.0 & 75.5 & 79.2 & 82.7 & 81.1 & 84.3 \\
\hline & 86.9 & 88.7 & 86.5 & 87.0 & 84.7 & 86.9 & 86.2 \\
\hline & 113.8 & 109.5 & 110.2 & 109.1 & 110.3 & 99.8 & 98.8 \\
\hline & 86.7 & 87.3 & 85.6 & 83.7 & 83.0 & 88.1 & 87.0 \\
\hline & 162.0 & 166.2 & 166.8 & 172.2 & 173.4 & 171.8 & 172.6 \\
\hline & 74.5 & 73.2 & 75.9 & 78.4 & 81.2 & 81.8 & 84.8 \\
\hline & 86.7 & 89.1 & 87.7 & 92.5 & 91.3 & 90.7 & 89.2 \\
\hline & 111.1 & 106.3 & 106.8 & 104.0 & 103.5 & 99.7 & 98.9 \\
\hline & 83.5 & 89.0 & 87.5 & 90.0 & 88.1 & 91.5 & 90.3 \\
\hline & 74.5 & 73.1 & 75.6 & 73.5 & 76.3 & 81.8 & 84.4 \\
\hline & 109.0 & 109.0 & 108.7 & 100.6 & 99.3 & 100.5 & 99.6 \\
\hline & 95.5 & 92.3 & 90.8 & 90.5 & 89.9 & 87.9 & 86.9 \\
\hline & 151.8 & 155.6 & 157.3 & 162.1 & 163.4 & 170.1 & 172.2 \\
\hline
\end{tabular}




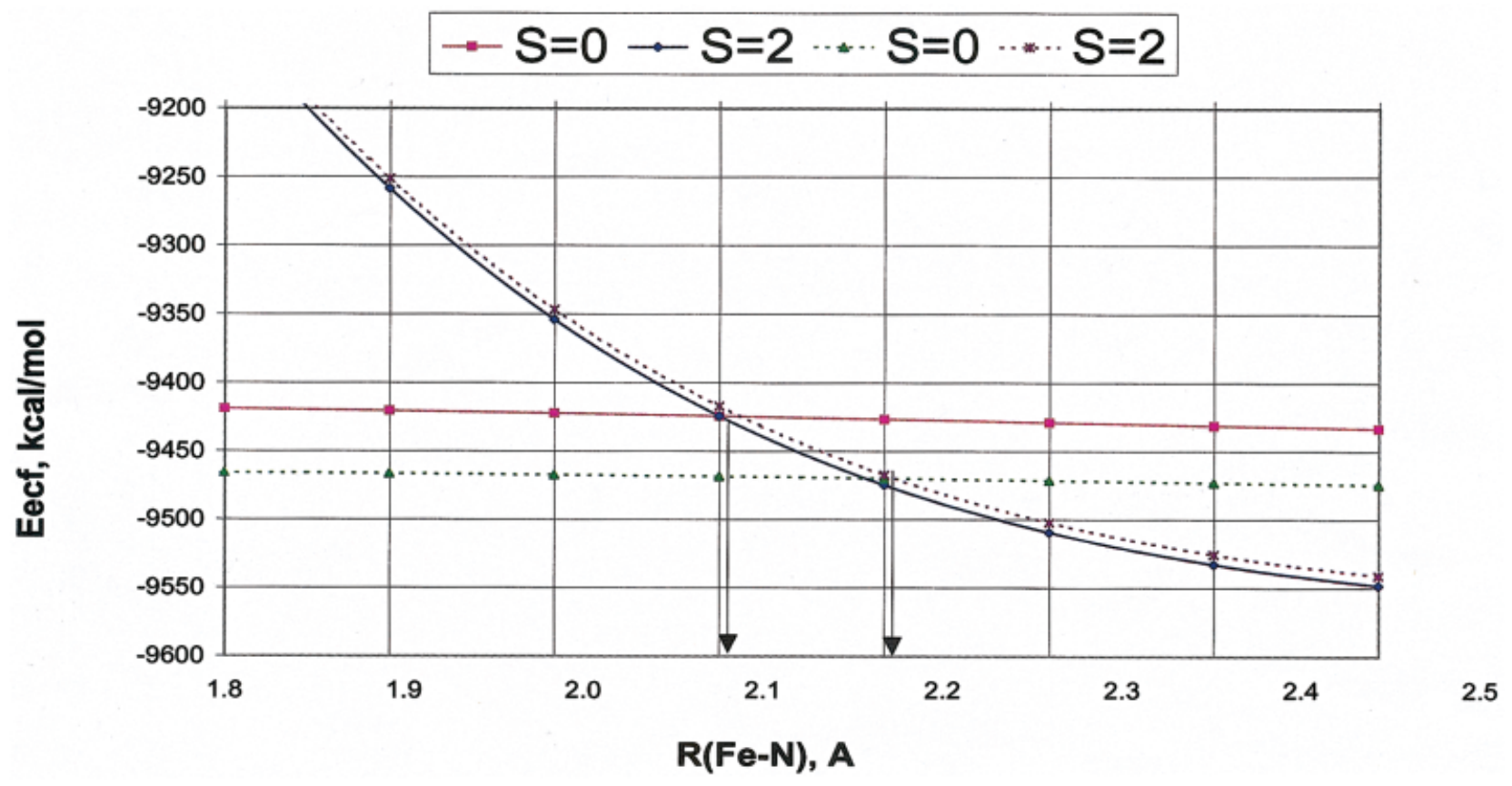

FIGURE 3. Dependence of the LECF-RLMO energy on the $\mathrm{Fe}-\mathrm{N}$ distance for the full symmetrical deformation of the $\mathrm{Fe}(\mathrm{Py}) 6$ complex. Dashed lines, $\eta=1$; solid lines, $\eta=1.5$. [Color figure can be viewed in the online issue, which is available at www.interscience.wiley.com.]

high-spin (triplet) six-coordinate and the low-spin (singlet) square planar four-coordinate complexes. By contrast, the (L)ECF method explicitly takes into account the electron correlation in the $d$-shell by employing the full configuration interaction wave function for the latter. It makes it possible to describe correctly the low-lying excited states of the $d$-shell and the crossovers that are required for description of the spin transitions [35, 48]. That allowed us to trace the effect of tiny geometry variations on the ground-state total spin in the complexes that maintain their octahedral geometry and the whole difference in geometry does not exceed $10 \%$ of the metal-ligand bond length.

\section{ACKNOWLEDGMENTS}

This work was performed with partial financial support of the Federal Target Program of Russia "Integracia" through Grant A0078. The usage of the CCSDB is supported by RFBR Grant 99-07-90133; use of the supercomputer facilities at the Keldysh Institute of Applied Mathematics is supported by RFBR Grant 01-07-90383.

\section{References}

1. Burkert, U.; Allinger, T. Molecular Mechanics; ACS: Washington, DC, 1982.

2. Brubaker, G. R.; Johnson, D. W. Coord Chem Rev 1984, 53, 1.

3. Allured, V. S.; Kelly, C. M.; Landis, C. R. J Am Chem Soc 1991, 113, 1.

4. Rappe, A. K.; Colwell, K. S.; Casewit, C. J Inorg Chem 1993, 32, 3438.

5. Sabolovic, J.; Raos, N.; Rasmussen, K. Croat Chem Acta 1989, 62(3), 495

6. Hambley, T. W.; Hawkins, C. J.; Palmer, J. A.; Snow, M. R. Aust J Chem 1981, 34, 45.

7. Reichert, D. E.; Hancock, R. D.; Welch, M. J Inorg Chem 1996, 35(24), 7013-7020.

8. Cundari, T. R.; Fu, W.; Moody, E. W.; Slavin, L. L.; Snyder, L. A.; Sommerer, S. O. J Phys Chem 1996, 100(46), 1805718064.

9. Johnson, D. W.; Brubaker, G. R. Inorg Chim Acta 1986, 119, 131.

10. Pletnev, I. V.; Melnikov, V. L. Koord Khim 1997, 23, 205 (in Russian).

11. Razumov, M. G.; Melnikov, V. L.; Pletnev, I. V. J Comput Chem 2001, 22(1), 38 .

12. Gillespie, R. J.; Hargittai, I. The VSEPR Model of Molecular Geometry; Allyn and Bacon, London, 1991.

13. Kepert, D. L. Inorganic Stereochemistry; Springer: Berlin, 1982. 
14. Darhovskii, M. B.; Tchougréeff, A. L. Chem Phys Rep 1999, 18,73 .

15. König, E.; Ritter, G.; Kulshreshtha, S. K. Chem Rev 1985, 85, 219.

16. Gütlich, P. Struct Bond 1981, 44, 83.

17. Tchougréeff, A. L. Chem Phys Rep 1997, 16, 62.

18. Tchougréeff, A. L. In Banci, L.; Comba, P., eds. Molecular Modeling and Dynamics of Bioinorganic Systems; NATO ASI workshop; Kluwer: Berlin, 1997, 217.

19. Burton, V.; Deeth, R.; Kemp, C.; Gilbert, P. J Am Chem Soc 1995, 117: 8407.

20. Deeth, R.; Paget, V. J Chem Soc Dalton Trans 1997, 537.

21. Gerloch, M.; Wooley, R. G. Struct Bond 1983, 46: 371.

22. Darhovskii, M. B.; Tchougréeff, A. L. Russ J Phys Chem 2000, $74,212$.

23. Soudackov, A. V.; Tchougréeff, A. L.; Misurkin, I. A. Theor Chim Acta 1992, 83, 389.

24. Tchougréeff, A. L. Phys Chem Chem Phys 1999, 1, 1051.

25. Lever, A. B. P. Inorganic Electronic Spectroscopy; Elsevier Science: Amsterdam, 1984.

26. Soudackov, A. V.; Tchougréeff, A. L.; Misurkin, I. A. Int J Quantum Chem 1996, 57, 663.

27. Soudackov, A. V.; Tchougréeff, A. L.; Misurkin, I. A. Int J Quantum Chem 1996, 58, 161.

28. Soudackov, A. V.; Tchougréeff, A. L.; Misurkin, I. A. Russ J Phys Chem 1994, 68, 1256.

29. Soudackov, A. V.; Tchougréeff, A. L.; Misurkin, I. A. Russ J Phys Chem 1994, 68, 1264.

30. Shaeffer, C. E. Struct Bond 1968, 5, 68.
31. Abrikosov, A. A.; Gor'kov, L. P.; Dzyaloshinskii, I. E. Methods of Quantum Field Theory in Statistical Physics, 2nd ed.; Dobrosvet: Moscow, 1998 (in Russian).

32. Thouless, D. J. The Quantum Mechanics of Many-Body System. Academic Press: New York, 1972.

33. Perkins, P. G.; Stewart, J. J. P. J Chem Soc Faraday Trans II 1982, 78, 285.

34. Stewart, J. J. P. J Comput Chem 1989, 10, 221.

35. Tchougréeff, A. L.; Soudackov, A. V.; Misurkin, I. A.; Bolvin, H.; Kahn, O. Chem Phys 1995, 193, 19.

36. Brooks, B. R.; Bruccoleri, R. E.; Olafson, B. D.; Dates, D. J.; Swaminathan, S.; Karplus, M. J Comput Chem 1983, 4, 187.

37. Smith, J. C.; Karplus, M. J Am Chem Soc 1992, 114, 801.

38. Di Sipio, L.; Tondello, E.; De Michelis, G.; Oleari, L. Chem Phys Lett 1971, 11, 287.

39. Böhm, M. C.; Gleiter, R. Theor Chim Acta 1981, 59, 127.

40. Pople, J. A.; Beveridge, D. L. Approximate Molecular Orbital Theory. McGraw-Hill: New York, 1970.

41. http:/ qcc.ru/ netlab: Entry ECFMM.

42. Nelder, J. A.; Mead, R. Comp J 1965, 7, 308.

43. Brent, R. P. Algorithms for Minimization Without Derivatives; Prentice Hall: Englewood Cliffs, NJ, 1973.

44. Doedens, R. J.; Dahl, L. F. J Am Chem Soc 1966, 88, 4847.

45. Onggo, D.; Hook, J. M.; Rae, A. D.; Goodwin, H. A. Inorg Chim Acta 1990, 173, 19.

46. Dick, S. Z. Kristallogr New Crystal Struct 1998, 213, 356.

47. Laine, P.; Gourdon, A.; Launay, J.-P. Inorg Chem 1995, 34, 5156. 